\title{
Associations between Body Composition, Disease Activity and Quality of Life in Patients with Crohn's Disease: A Cross-Sectional Study
}

\author{
Hilligsøe $\mathbf{M}^{1}$, Eriksen $\mathrm{ND}^{1}$, Jacobsen $\mathrm{MH}^{1}$, Kaagaard $\mathrm{LH}^{1}$, Rasmussen $\mathrm{HH}^{2}$, Vinter-Jensen $\mathrm{L}^{2}$ and $\mathrm{Holst}^{2}{ }^{2 *}$ \\ ${ }^{1}$ Department of Health Sciences, Aalborg University, Denmark \\ ${ }^{2}$ Department of Gastroenterology and Department of Clinical Medicine, Aalborg University Hospital and Aalborg University, Denmark
}

Submission: March 12, 2019; Published: April 22, 2019

"Corresponding author: Mette Holst, Head of Clinical Nutrition Research, Center for Nutrition and Bowel Disease, Department of Gastroenterology, Aalborg University Hospital, and Department of Clinical Medicine, Aalborg University, Aalborg Moelleparkvej 4, 2 Reception 39000 Aalborg, Denmark

Abstract

Background \& Aims: Patients with Crohn's disease (CD) treated with tumor necrosis factor- $\alpha$ (TNF- $\alpha$ ) inhibitors are heterogeneous and respond differently to treatment. We aimed to investigate if disease activity and quality of life were associated with the individual varying distribution of fat and fat free mass.

Methods: A sample of 83 CD patients treated with TNF- $\alpha$ inhibitors were examined in this single center, cross-sectional study. Body composition was measured by bioelectrical impedance analysis (BIA) and muscle function by handgrip strength (HGS). The association to disease activity assessed by Harvey-Bradshaw Index (HBI), C- reactive protein (CRP) and quality of life assessed by Short Health Scale (SHS) was investigated.

Results: Increased fat mass index (FMI) was significantly associated to higher disease activity assessed by HBI $(\mathrm{p}<0.05)$ and showed a trend toward a higher CRP level in multivariate non-parametric regression analyses ( $p=0.07)$. Univariate tests showed a significant positive association between FMI and SHS as well. Fat free mass index (FFMI) and HGS were not associated to disease activity or quality of life in this population.

Conclusion: Despite TNF- $\alpha$ inhibitor therapy, one third of the patients in this study experienced active disease. High FMI was significantly associated to increased disease activity and decreased quality of life. No associations were seen between FFMI, HGS and body composition. Larger studies are needed to confirm these results, and to investigate confounders.

Keywords: Crohn's disease; Tumor necrosis factor alpha; Antagonists and inhibitors; Body composition; Disease activity; Quality of life; Adalimumab; Bioelectrical impedance analysis; Fat free mass index; Fat mass index; Infliximab; Pro-inflammatory cytokine; Adipocytes; Mesenteric fat; Skeletal muscle mass

Abbrevations: AAUH: Aalborg University Hospital; ADA: Adalimumab; BIA: Bioelectrical Impedance Analysis; BMI: Body Mass Index; CD: Crohn's Disease; FFMI: Fat Free Mass Index; FMI: Fat Mass Index; HBI: Harvey-Bradshaw Index; HGS: Handgrip Strength; IFX: Infliximab; SHS: Short Health Scale; TNF- $\alpha$ : Tumor Necrosis Factor Alpha

\section{Introduction}

Crohn's disease (CD) is a chronic inflammatory bowel disease, which is characterized by discontinuing, transmural, inflammatory lesions, which can affect any part of the gastrointestinal tract. Tumor necrosis factor- $\alpha$ (TNF- $\alpha$ ) is a pro-inflammatory cytokine and is often increased in patients with CD compared to healthy populations. The blockage of the TNF- $\alpha$ pathway using TNF- $\alpha$ inhibitors like Infliximab (IFX) and Adalimumab (ADA) has shown efficacy in treatment of CD [1]. However, between 23-46\% of all patients do not respond sufficiently to treatment with TNF- $\alpha$ inhibitors 12 months after initiation [2].
Adipocytes, especially those in mesenteric fat, have shown to be a source of TNF- $\alpha$ and there seem to be a correlation between distribution of fat tissue, the production of TNF- $\alpha$ and activity of disease [3]. A few studies have shown high body mass index (BMI) and body fat levels to be associated with reduced response to IFX [4] and that patients with high fat ratios required increased dosage of ADA [5].

Muscle mass has also been shown to affect progress of disease. In one study, high muscle mass ratio before surgery was correlated 
to less postoperative complications [6]. Furthermore, loss of skeletal muscle mass has shown to be associated with poor response to TNF- $\alpha$-inhibitor treatment $[7,8]$.

Body composition parameters like fat and muscle mass are often altered in patients with $\mathrm{CD}$ [9], which might be explained by the symptoms of disease (diarrhea, reduced nutrition absorption etc.), the catabolic role of inflammation and by the frequent use of corticosteroids $[1,10]$. Especially weight- and muscle loss during disease activity are often seen. Treatment with TNF- $\alpha$ inhibitors have shown to improve muscle parameters in the same group of patients $[1,11]$.

These findings give reason to investigate in further details, whether disease activity and quality of life are associated with the distribution of fat and muscle mass, which was the aim of this study.

\section{Materials and Methods}

\section{Sampling}

During a five-week recruitment period in 2017, 127 CD patients in IFX or ADA treatment were invited to participate in this cross-sectional study at Aalborg University Hospital (AAUH). Only patients in maintenance IFX or ADA therapy were included. Patients in induction regimes (first 8 weeks after initiation) were excluded. Exclusion criteria further consisted of pregnancies, limb prostheses, implanted electronic devices and inability to stand up, according to the bioelectrical impedance analysis (BIA) measurement guidelines.

\section{Measurement of body composition and muscle strength}

Body composition was measured by BIA and performed with Tanita Body Composition Analyzer BC-418MA (Tanita corporation, Japan). Data gathered from BIA included BMI $(\mathrm{kg} / \mathrm{m} 2)$, fat mass index (FMI) $(\mathrm{kg} / \mathrm{m} 2)$ and fat free mass index (FFMI) $(\mathrm{kg} /$ $\mathrm{m} 2$ ). Patients were instructed to fast at least 2 hours and to pass water latest 30 minutes before the BIA measurement.

Hand grip strength (HGS), which is a functional objective correlated to muscle mass, was measured with SAEHAN ${ }^{\circledR}$ DHD-1 Digital Hand Dynamometer (SAEHAN corporation, South. Korea, 2013) [12]. Patients were encouraged to apply maximal power with their dominant hand 3 times with 15 seconds of rest in between. The maximal value for each patient was included in the study.

\section{Assessment of disease activity and quality of life}

As a standard approach to TNF- $\alpha$-inhibitor therapy at AAUH, patients with CD complete a Harvey Bradshaw Index (HBI) and Short Health Scale (SHS) questionnaire on touch screens prior to treatment. These scores together with information about treatment status and blood sample values (CRP, albumin, hemoglobin) are collected and stored in "Gastrobio", a database developed by Department of Gastroenterology and Hepatology, AAUH [13]. HBI and SHS were used as subjective assessments of disease activity and quality of life, while CRP was used as an objective measure- ment for disease activity. High values of HBI and CRP indicate more severe disease activity and high values of SHS indicate reduced quality of life. HBI $\geq 5$ indicates active disease [14]

\section{Statistical analysis}

Results are presented as means with standard deviations (SD) for parametric data and as medians with interquartile ranges (IQR) for nonparametric data. Nominal data are presented as numbers (\%). Uni- and multivariable non-parametric regression analyses were used to investigate the associations between disease activity (HBI and CRP), quality of life (SHS), body composition parameters (BMI, FMI, FFMI) and muscle function (HGS).

Bootstrapping using 1000 replications were used to construct 95\% percentile confidence intervals (CI) for multivariable estimates. A p-value $<0.05$ was considered statistically significant. The software package STATA version 15.1 (Stata Corp LP, College Station, Texas) was used for statistical calculations [15].

\section{Ethical considerations}

This study did not cause any interventions to the current therapy standards or expend of biological material. Thus, the ethical committee of North Jutland Region had no objections to the study. The Danish data protection agency was notified about the study. Patients gave verbal and written consents prior to the study.

\section{Results}

\section{Patient characteristics}

Table 1: Patient characteristics for the total population, $n=83$

\begin{tabular}{|c|c|}
\hline Demographic Information & \\
\hline Gender (men), n (\%) & $39(47.0 \%)$ \\
\hline Age, years (SD) & $37.3(12.6)$ \\
\hline Smoker, n (\%) & $19(22.9 \%)$ \\
\hline \multicolumn{2}{|c|}{ Activity of Disease } \\
\hline Disease duration, years (SD) & $11.2(9.5)$ \\
\hline HBI, median (IQR) & $3(1-5)$ \\
\hline $\mathrm{HBI} \geq 5, \mathrm{n}(\%)$ & $25(30.1 \%)$ \\
\hline SHS, median (IQR) & $25(12-48)$ \\
\hline CRP, mg/L (SD) n=75 & $5.6(12.2)$ \\
\hline \multicolumn{2}{|c|}{ Treatment Status } \\
\hline Infliximab, n (\%) & $58(70 \%)$ \\
\hline Adalimumab, n (\%) & $25(30 . \%)$ \\
\hline Treatment duration, years (SD) & $5.3(4.0)$ \\
\hline \multicolumn{2}{|l|}{ Combination therapy } \\
\hline Azathioprine/Methotrexate, n (\%) & $43(51.8 \%)$ \\
\hline Corticosteroids, n (\%) & $6(7.2 \%)$ \\
\hline \multicolumn{2}{|c|}{ Body Composition } \\
\hline Weight, kg (SD) & $76.9(14.1)$ \\
\hline BMI, kg/m² (SD) & $25.4(4.5)$ \\
\hline Fat mass index, $\mathrm{kg} / \mathrm{m}^{2}(\mathrm{SD})$ & $7.5(3.8)$ \\
\hline Fat free mass index, $\mathrm{kg} / \mathrm{m}^{2}$ (SD) & $17.9(2.3)$ \\
\hline Handgrip strength, $\mathrm{kg}(\mathrm{SD}) \mathrm{n}=82$ & $42.7(11.3)$ \\
\hline
\end{tabular}


In total 127 patients were invited during the inclusion period. Seven were excluded according to the BIA measurement guidelines. Out of the remaining 120 patients, 83 patients (69\%) with a mean age of $37.3( \pm 12.6)$ years accepted to participate and successfully completed the examination. Of these, 39 were men (47 $\%)$. Mean duration of disease and treatment were $11.2( \pm 9.5)$ and $5.2( \pm 4.0)$ years respectively. Of all patients, 25 (30.1\%) were in active disease according to $\mathrm{HBI} \geq 5$. Median HBI was 3 (IQR 1-5), mean CRP was $5.58( \pm 12.2) \mathrm{g} / \mathrm{L}$ and median SHS was 25 (IQR 1248). Mean values for body composition parameters were BMI 25.4 $( \pm 4.5 \mathrm{~kg} / \mathrm{m} 2)$, FMI $7.5( \pm 3.8) \mathrm{kg} / \mathrm{m} 2$, FFMI $17.9( \pm 2.3) \mathrm{kg} / \mathrm{m} 2$ and HGS 42.7 ( \pm 11.3 ) kg. Full list of main characteristics of the study population is presented in Table 1 .

\section{Associations between body composition, disease activity and quality of life}

Table 2: Non-parametric regression analysis between body composition (BMI, FMI, FFMI and HGS), disease activity (HBI and CRP) and quality of life (SHS).

\begin{tabular}{|c|c|c|c|c|c|c|}
\hline & \multicolumn{2}{|c|}{ HBI } & \multicolumn{2}{c|}{ CRP } & \multicolumn{2}{c|}{ SHS } \\
\hline & Coefficient & $\mathrm{p}$-Value & Coefficient & $\mathrm{p}$-Value & Coefficient & 0.98 \\
\hline BMI & 0.29 & 0.01 & 0.31 & 0.17 & 1.27 & 0.05 \\
\hline Fat Mass Index & 0.42 & 0.004 & 0.6 & 0.07 & 0.06 & 0.95 \\
\hline $\begin{array}{c}\text { Fat Free Mass } \\
\text { Index }\end{array}$ & 0.09 & 0.74 & -0.56 & 0.73 & 0.26 & 0.31 \\
\hline HGS & -0.01 & 0.89 & -0.13 & 0.23 & 0.96 \\
\hline
\end{tabular}

Univariate non-parametric regression analyses were used to investigate any associations between disease activity, quality of life and body composition parameters. Table 2 shows associations. In our population high values of BMI were associated with significantly higher scores on HBI (coefficient $0.29, \mathrm{p}=0.01$ ) and SHS (coefficient 0.98, p 0.05) indicating more severe disease acsociated with significantly higher scores on HBI (coefficient 0.042 , $\mathrm{p}=0.004$ ) and SHS (coefficient 1.27, $\mathrm{p}=0.04$ ), indicating the same pattern. Further, a trend between high values of FMI and CRP was observed, but did not show any significance (coefficient 0.60 , $\mathrm{p}=$ 0.07). FFMI and HGS were not associated to body composition parameters. tivity and less quality of life. Likewise, high values of FMI were as-

Table 3: Multivariate analysis of associations between body composition (BMI, FMI, FFMI and HGS), disease activity (HBI and CRP) and quality of life (SHS). ${ }^{*} 95 \%$ confidence intervals $(95 \% \mathrm{Cl})$ and $\mathrm{p}$-values were calculated using bootstrap-corrected analysis.

\begin{tabular}{|c|c|c|c|c|c|c|}
\hline & \multicolumn{2}{|c|}{ HBI } & \multicolumn{2}{c|}{ CRP } & \multicolumn{2}{c|}{ SHS } \\
\hline & Coefficient $(95 \% \mathrm{CI})^{*}$ & $\mathrm{p}$-Value & Coefficient $(95 \% \mathrm{CI})^{*}$ & $\mathrm{p}$-Value & Coefficient $(95 \% \mathrm{CI})^{*}$ & $\mathrm{p}$-Value \\
\hline BMI & $-0.04(-0.38$ to 0.33$)$ & 0.82 & $-0.58(-1.51$ to 0.21$)$ & 0.19 & $0.22(-1.67$ to 2.62$)$ & 0.84 \\
\hline Fat mass index & $0.46(0.08$ to 0.86$)$ & 0.02 & $1.21(-0.01$ to 2.56$)$ & 0.07 & $1.04(-1.68$ to 3.15$)$ & 0.41 \\
\hline
\end{tabular}

Multivariate non-parametric regression analyses performed between body composition parameters (BMI, FMI, FFMI and HGS) and the outcome variables (HBI, CRP and SHS) confirmed the significant association between FMI and HBI (coefficient 0.46, p= 0.02 ) and the non-significant trend between FMI and CRP (coefficient $1.21, \mathrm{p}=0.07$ ). Conversely, no significance was seen between BMI, SHS and HBI. Neither did SHS and FMI show significance in multivariate regression analysis. See Table 3.

\section{Discussion}

This study aimed to investigate the associations between body composition, disease activity and quality of life in CD patients treated with TNF- $\alpha$ inhibitors. Multivariate analysis found FMI to be significantly associated with disease activity assessed by HBI and in univariate analysis to be significantly associated with quality of life assessed by SHS. BMI was significantly associated to HBI and SHS by univariate analyses, but not in multivariate analysis. FFMI and HGS showed no associations to neither disease activity nor quality of life in this study.

One third ( $n=25,30.1 \%$ ) of all patients included in this study scored $\mathrm{HBI} \geq 5$, indicating signs of active disease. This prevalence corresponds with the findings from a review by Ben-Horin et al. [2] which reports primary non-response to occur in 10-40\% and secondary non-response to occur in $23-46 \%$ of all patients treated with TNF- $\alpha$-inhibitors.

FMI and BMI were significantly associated to disease activity in the form of HBI in univariate analysis, but only FMI showed to be significant in multivariate analysis. This could be explained by the fact that BMI is highly affected by FMI and therefore showed no independent significance.

High BMI and body fat have earlier been associated to poor response to IFX treatment and fatty tissues have shown to be a source of pro-inflammatory cytokines such as TNF- $\alpha$ and CRP $[3,4]$. Peyrin-Biroulet et al. [16] further found mesenteric fat as an important source of CRP-elevation in $\mathrm{CD}$ and that the local production of CRP from mesenteric adipocytes might be stimulated by local inflammation in CD. Increased body fat and from this, increased mesenteric fat, could be an explanation to the increased disease activity assessed by HBI in the more obese patients in this study. This association between high fat mass and inflammation has been shown in former studies as well $[5,17,18]$. 
An eventual poor response to TNF- $\alpha$-inhibitor therapy in patients with increased FMI besides the possible pro-inflammatory role of fat, could be the distribution of drug into fat tissues leading to a larger volume of distribution and thus lower drug concentration. IFX is administered intravenously, and Scaldaferri et al. [4] suggest that IFX does not distribute into adipose tissue. ADA on the other hand is administered subcutaneously and pharmacokinetics could be altered in the more obese patients $[5,19]$.

CRP was used as an objective measurement of disease activity and showed a non-significant trend towards higher FMI in univariate and multivariate test. This might indicate that high FMI was not only associated to higher $\mathrm{HBI}$, which is a subjective assessment of disease activity, but also to objective measurements of disease activity like CRP. Despite no significance, disease activity was negatively associated to FFMI and HGS pointing towards increased FFMI being a protective factor against increased disease activity as suggested by Zhang et al. [6] However, the lack of significance may be due to the relatively low sample size in this study.

Stjernman et al. [20] have concluded that SHS is a valid, reliable and responsive tool to quantify health related quality of life in $\mathrm{CD}$ patients. No investigations have been made regarding the relationship between body composition and health related quality of life in CD patients. In our findings, SHS was only significantly associated to FMI in univariate analysis, indicating a high FMI associated to reduced quality of life. Multivariate analysis showed no significance, making the association unclear and a topic for further investigation.

In this study, body composition was analyzed by the use of BIA, which is a cheap, non-invasive and fairly sensitive tool. However, other methods like DEXA, CT and MRI scans are more accurate and provide direct measurement of fat mass and fat free mass including the distinction between mesenteric and subcutaneous fat levels $[8,11]$. The BIA equipment used in this study did not allow to distinguish between different types of fat and no further investigations into the pro-inflammatory role of mesenteric fat could be made. The benefit of CT and MRI scans is that many patients have gone through a CT or MRI scan as part of their treatment of CD, which provides the opportunity for a retrospective study design investigating the associations between disease activity, body composition and quality of life in a larger population.

\section{Conclusion}

The purpose of this cross-sectional study was to explore if body composition in patients with CD treated with TNF- $\alpha$ inhibitors was associated to disease activity and quality of life. We found that almost one third of the patients showed signs of active disease, despite TNF- $\alpha$ inhibitor treatment. High FMI showed a significantly higher disease activity assessed by HBI and a trend towards higher CRP. This could indicate a pro-inflammatory role of fat and should be investigated further. FMI was also associated to poorer quality of life assessed by SHS, but only in univariate tests indicating more factors to influence this association as well.
FFMI and HGS were not associated to neither disease activity nor quality of life.

\section{Acknowledgements}

Mads Hilligsøe, Niklas D Eriksen, Maria H Jacobsen, Liv H Kaagaard and Mette Holst were responsible for the study design, data collection and analysis, drafted the manuscript and wrote the final edition. Henrik H Rasmussen and Lars Vinter-Jensen provided scientific guidance and medical expertise, contributed to the analysis and critically reviewed the manuscript.

The authors would like to thank participants, Tove Nygaard, Lotte Julin Hansen at Department of Gastroenterology, AAUH for providing the logistics and Department of Health Information, AAUH for sharing their facilities. A special thanks to Lone Larsen, Consultant MD, PhD stud., for extensive help regarding the Gastrobio database.

\section{References}

1. Csontos ÁA, Molnár A, Piri Z, Katona B, Dakó S, et al. (2016) The Effect of anti-TNF $\alpha$ induction therapy on the nutritional status and dietary intake in inflammatory bowel disease. J Gastrointest Liver Dis 25(1): 49-56.

2. Ben-Horin S, Kopylov U, Chowers Y (2014) Optimizing anti-TNF treatments in inflammatory bowel disease. Autoimmun Rev 13(1): 2430.

3. Popko K, Gorska E, Stelmaszczyk-Emmel A, Plywaczewski R, Stoklosa A, et al. (2010) Proinflammatory cytokines Il- 6 and TNF- $\alpha$ and the development of inflammation in obese subjects. Eur J Med Res 15(Suppl 2): 120-122.

4. Scaldaferri F, D’Ambrosio D, Currò D, Petito V, Pecere S, et al. (2015) Role of BMI, use of immune suppressant and pharmacokinetic of infliximabpathway in determining prospective and retrospective response to the drug in cohort of IBD patients under maintenance therapy with infliximab. Dig Liver Dis 12(10): e0186575.

5. Bultman E, De Haar C, Van Liere-Baron A, Verhoog H, West RL, et al (2012) Predictors of dose escalation of adalimumab in a prospective cohort of Crohn's disease patients. Aliment Pharmacol Ther 35(3): 335-341.

6. Zhang W, Zhu W, Ren J, Zuo L, Wu X, et al. (2015) Skeletal Muscle Percentage: A Protective Factor for Postoperative Morbidity in Crohn's Disease Patients with Severe Malnutrition. J Gastrointest Surg 19(4): 715-721.

7. Holt DQ, Varma P, Strauss BJG, Rajadurai AS, Moore GT (2015) Low muscle mass at treatment initiation is associated with early loss of response to anti-TNF therapy for Inflammatory Bowel Disease. J Gastroenterol Hepatol 30: 131.

8. Ding NS, Malietzis G, Lung PF, Yip W, Penez L (2016) Body composition: A biomarker of therapeutic outcome in patients with Crohn's disease. J Crohn's Colitis. N.S. Ding, St Mark's Hospital, IBD, London, United Kingdom, 10: S402-403.

9. Back IR, Marcon SS, Gaino NM, Salate D, Vulcano B, et al. (2017) Body composition in patients with Crohn's disease and ulcerative colitis. Arq Gastroenterol 54(2): 109-114.

10. Wiroth JB, Filippi J, Schneider SM, Al-Jaouni R, Horvais N, et al. (2005) Muscle performance in patients with Crohn's disease in clinical remission. Inflamm Bowel Dis 11(3): 296-303.

11. Emerenziani S, Biancone L, Guarino MPL, Balestrieri P, Stasi E, et al. (2016) Nutritional status and bioelectrical phase angle assessment in 
adult Crohn disease patients receiving anti-TNF $\alpha$ therapy. Dig Liver Dis 49(5): 495-499.

12. Lu ZL, Wang TR, Qiao YQ, Zheng Q, Sun Y, et al. (2016) Handgrip Strength Index Predicts Nutritional Status as a Complement to Body Mass Index in Crohn's Disease. J Crohn's Colitis 10(12): 1395-1400.

13. Larsen L, Drewes AM, Fallingborg J, Jacobsen BA, Jess T (2016) Touch screens as a tool in patient care in the IBD outpatient clinic. Scand J Gastroenterol 51(9): 1106-1110.

14. (2010) Inc. J. Harvey-Bradshaw Index (HBI) - A simple index of Crohn's disease activity. British Columbia Ministry of Health Services. Worksheet based on the Harvey-Bradshaw Index. British Columbia Ministry of Health Services website.

15. Brandse JF, Vos LMC, Jansen J, Schakel T, Ponsioen CIJ, et al. (2015) Serum concentration of Anti-TNF antibodies, adverse effects and quality of life in patients with inflammatory bowel disease in remission on maintenance treatment. J Crohn's Colitis 9(11): 973-981.
16. Peyrin-Biroulet L, Gonzalez F, Dubuquoy L, Rousseaux C, Dubuquoy C et al. (2012) Mesenteric fat as a source of $C$ reactive protein and as a target for bacterial translocation in Crohn's disease. Gut 61(1): 78-85.

17. Ordás I, Feagan BG, Sandborn WJ (2012) Therapeutic Drug Monitoring of Tumor Necrosis Factor Antagonists in Inflammatory Bowel Disease. Clin Gastroenterol Hepatol 10(10): 1079-1087.

18. Yarur AJ, Abreu MT, Deshpande AR, Kerman DH, Sussman DA (2014) Therapeutic drug monitoring in patients with inflammatory bowel disease. World J Gastroenterol 20(13): 3475-3484.

19. Hanley MJ, Abernethy DR, Greenblatt DJ (2010) Effect of obesity on the pharmacokinetics of drug in humans. Clin pharmacokinet 49(2): 7187.

20. Stjernman H, Granno C, Jarnerot G, Ockander L, Tysk C, et al. (2008) Short health scale: A valid, reliable, and responsive instrument for subjective health assessment in Crohn's disease. Imflamm Bowel Dis 14(1): 47-52. 